\title{
Measurement of standing position in door opening movement for fall prevention
}

\author{
Kaori Sato (Faculty of Health Science, Kinjo University, satokao@kinjo.ac.jp) \\ Masanobu Igarashi (Department of Rehabilitation, Tulip Nagae Hospital, crossgame-@docomo.ne.jp) \\ Yuki Igarashi (Department of Rehabilitation, Kitagawa Orthopedic Clinic, naymakmnhnhrmysmm@ezweb.ne.jp) \\ Takumi Nakamura (Department of Rehabilitation, Uno Hospital, info@uno.or.jp) \\ Yumi Osawa (Laforet fitness Salon, Sports Programs, yzrtbs@gmail.com)
}

\begin{abstract}
A door opening/closing movement is one of the necessary motions in the activity of daily living. In this study, we investigated the factors that affect the standing position of a door opening movement. Ninety-three young healthy volunteers (58 female and 35 male) participated in this study. We recorded the door opening/ closing movement using a GoPro Hero $4^{\mathbb{B}}$ camera from the ceiling under the following conditions. A hinged or sliding door was placed on a walkway with or without a simulated wall next to the door. After some images were taken from the camera, the distance between the great toe of the former foot and the door/wall (TD/TW distance) was calculated. The relationship among TD/TW distance and physical features were analysed by using multiple regression analyses. There were significant differences between TD and TW distance depending on door type, and wall presence showed a significant difference in TW distance. TW distance and the height were significant predictors of TD distance. TD distance was a significant predictor of TW distance. The result showed that the surrounding environment and the physical features affected the door opening movement.
\end{abstract}

\section{Keywords}

door opening movement, hinged door, sliding door, standing position, fall prevention

\section{Introduction}

A door opening movement is one of the necessary movements in the activities of daily life, and this movement is repeated many times every day. There are two main types of doors: sliding doors and open doors, each of which is used in various facilities. It could be said that the sliding door is recommended in the case of housing renovation because body moving would be minimized for opening/closing the door [Kurioka and Funakawa, 2004]. However, air-tightness is better with the opening door, and each door is considered to have advantages and disadvantages.

In a public facility, it is possible to omit the opening movement by using an automatic door. However, in places where water is used, it might be difficult to install an automatic door. Moreover, in an old building, major modification is required to receive a power supply, so there are many cases where it becomes difficult to install an automatic door. There are many hot springs and old hot spring villages, etc. in Japan, there are over 3,000 hot spring resorts and more than 12,000 accommodation facilities [Japanese Ministry of the Environment]. Hot springs are not only used in various ways for Japanese people's recreation and sightseeing, but it is also said that hot spring tourism plays an important role in the development and formation process of the tourist area [Yamada and Miyazaki, 2008]. In such a hot spring facility, the water is used, and in many cases, it is an old building, the door opening movement is considered to be one of the indispensable movements.

The door opening movement includes multiple phases of movements; walking close to the door, stopping, pushing/pulling the handle, and walking through the door. Therefore, these multicomplex movements might cause falling for elderly people and people with disabilities. Also, it is not well known that differences between types of door (hinged door/sliding door), and with or without the wall next to the door affect the distance between the human and the door/wall. It is still little known if the parameters including the height, upper and lower extremity length affect the distance between the former foot and the door. Physical features like height, body weight, trunk length, upper and lower extremity length affect dynamic balance [Okuma et al., 2001], but it was not discussed enough about the door opening movement.

The purpose of this study was to investigate the factors that affect the distance between the former foot and door/wall, also the physical features that affect the door opening movement.

\section{Subjects and method \\ 2.1 Subjects}

Ninety-three young healthy volunteers (58 female and 35 male) participated in this study. Persons with visual impairments and orthopaedic diseases were excluded. Because there were a few left-handed people in the pre-survey, only righthanded were included in this study. Average age of subjects was $21.1 \pm 0.2$ years. Each subject participated with informed consent in this study.

\subsection{Method}

Each subject's height and length of upper and lower extremity were measured by the 3rd and 5th authors. The upper extremity length was defined as the distance from the acromion of the scapula to the styloid process of the radius. The lower 
extremity length was defined as the distance from the anterior superior iliac spine (ASIS) of the ilium to the medial malleolus of the tibia.

GoPro Hero $4^{\circledR}$ cameras were used in this study to shoot the door opening movement. We placed $5 \mathrm{~cm}$ square grid paper on the floor in front of the door and set the camera on the ceiling to shoot the grid paper. We recorded the subjects walking on the grid paper. The types of the door we designed were a hinged and a sliding door which were opened to the right side. We placed a simulated wall next to the door. The experimental conditions were: (1) a hinged door with wall, (2) a hinged door without wall, (3) a sliding door with wall, and (4) a sliding door without wall. We recorded the movies three times in each condition and the order of conditions was randomized. Because the combination of the hand holding the door handle and former foot were not same in each trial, all trials were taken into account.

The lens distortion of the recorded movies was corrected by using the software (GoPro studio ver2.5). We checked the hand holding the door handle and former foot. Then the still images of opening door were taken from recorded movies to calculate the distance between the great toe of the former foot and door/ wall (TD/TW distance). The definition of TD/TW distance

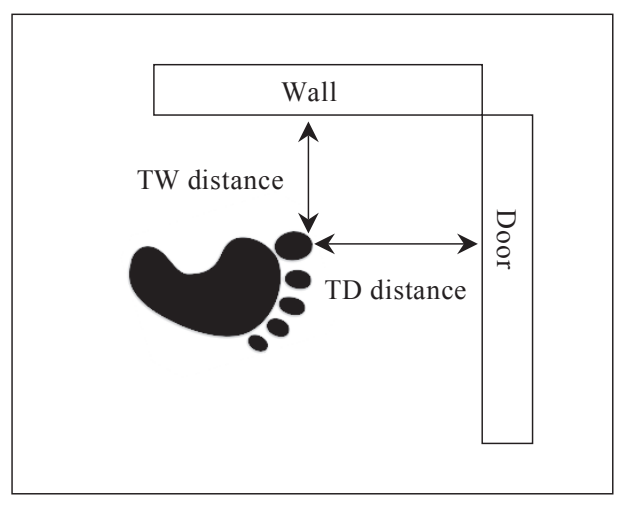

Figure 1: Definitions of TD/TW distance is shown in Figure 1. We measured the numbers of pixels in TD/TW distance and $10 \mathrm{~cm}$ on the grid paper using Image $\mathrm{J}$ ver.1.48. Then we calculated how many centimeters the TD/ TW distance were statistically significant.

Statistical analyses were carried out using IBM SPSS Statistic (Version 25.0). The relationship among TD/TW distance and physical features were analyzed by using the two-way ANOVA and the multiple regression analysis. A value of $p<$ 0.05 was considered.

\section{Results}

\subsection{Physical features of the subjects}

Average height of the subjects was $164.0 \pm 6.6 \mathrm{~cm}$ (male: $170.2 \pm 4.8 \mathrm{~cm}$, female: $160.3 \mathrm{~cm} \pm 4.3 \mathrm{~cm}$ ). Average length of upper extremity was $52.6 \pm 2.9 \mathrm{~cm}$ (male: $54.8 \pm 2.4 \mathrm{~cm}$, female: $51.4 \pm 2.2 \mathrm{~cm}$ ). Average height of the subjects was $82.5 \pm$ $5.3 \mathrm{~cm}$ (male: $85.0 \pm 6.3 \mathrm{~cm}$, female: $81.1 \pm 4.0 \mathrm{~cm}$ ).

\subsection{TD distance and TW distance}

Under the combination of each condition and the hand holding the door handle, former foot is shown in Table1.

The TD distance and TW distance are shown in Table 3. For the TD distance, a two-way ANOVA showed a statistical difference in door type $(p<0.001)$, but not wall presence. No interaction was detected between door type and wall presence. For the TW distance, a two-way ANOVA showed a statistical difference in door type $(p<0.001)$, and wall presence $(p<$ $0.001)$. No interaction was detected between door type and wall presence.

A multiple regression analysis was performed to predict TD distance based on TW distance, the length of upper and lower extremity and height (Table 4). Two regression equations were created, and the TW distance and height were chosen as explanatory variables.

A multiple regression analysis was also performed to predict TW distance based on TD distance, the length of upper and lower extremity and height (Table 5). One regression equation

Table 1: Number of the trials under hinged door condition

\begin{tabular}{lcccccccccc}
\hline & \multicolumn{1}{c}{ Hinged Door $(N=558)$} \\
\hline Wall presence & \multicolumn{3}{c}{ With } & \multicolumn{7}{c}{ Without } \\
\hline Hand holding the door handle & \multicolumn{2}{c}{ R } & & & L & & R & & L \\
\hline Former foot & R & L & R & L & R & L & R & L \\
\hline Number of trials & 83 & 169 & 15 & 12 & 92 & 163 & 17 & 7 \\
\hline
\end{tabular}

Table 2: Number of the trials under sliding door condition

\begin{tabular}{lccccccccccc}
\hline & \multicolumn{1}{c}{ Sliding Door $(N=558)$} \\
\hline Wall presence & \multicolumn{4}{c}{ With } & \multicolumn{7}{c}{ Without } \\
\hline Hand holding the door handle & \multicolumn{1}{c}{ R } & & & L & & & R & & L \\
\hline Former foot & R & L & R & L & R & L & R & L \\
\hline Number of trials & 121 & 109 & 24 & 25 & 95 & 143 & 20 & 21 \\
\hline
\end{tabular}


Table 3: The TD distance and TW distance

\begin{tabular}{lcccc}
\hline & \multicolumn{2}{c}{ Hinged Door $(N=558)$} & \multicolumn{2}{c}{ Sliding Door $(N=558)$} \\
\hline Wall presence & With & Without & With & Without \\
\hline TD distance $(\mathrm{cm})$ & $34.0 \pm 15.9$ & $35.1 \pm 16.1$ & $18.9 \pm 8.4$ & $18.0 \pm 8.5$ \\
\hline TW distance $(\mathrm{cm})$ & $31.0 \pm 12.9$ & $28.7 \pm 12.6$ & $27.3 \pm 7.7$ & $25.0 \pm 8.4$ \\
\hline
\end{tabular}

Table 4: Result of multiple-linear regression in TD distance

\begin{tabular}{|c|c|c|c|c|c|c|}
\hline & \multirow{2}{*}{ Item } & \multirow{2}{*}{$\begin{array}{l}\text { Partial regression } \\
\text { coefficient }\end{array}$} & \multirow{2}{*}{$\begin{array}{l}\text { Standardized partial } \\
\text { regression coefficient }\end{array}$} & \multirow{2}{*}{$p$ value } & \multicolumn{2}{|c|}{$95 \%$ Confidence interval } \\
\hline & & & & & Lower bound & Upper Bound \\
\hline \multirow{3}{*}{1} & Constant & 19.507 & & $p=0.000$ & 17.096 & 21.918 \\
\hline & TW Distance & 0.251 & 0.180 & $p<0.001$ & 0.170 & 0.331 \\
\hline & \multicolumn{6}{|c|}{$\left(\mathrm{R}^{2}=0.033\right.$, Analysis of Variance: $\left.p<0.01\right)$} \\
\hline \multirow{4}{*}{2} & Constant & -9.693 & & $p=0.382$ & -31.422 & 12.037 \\
\hline & TW Distance & 0.248 & 0.178 & $p<0.001$ & 0.167 & 0.328 \\
\hline & Hight & 0.179 & 0.078 & $p<0.008$ & 0.046 & 0.311 \\
\hline & $\left(\mathrm{R}^{2}=0.039, \mathrm{~A}\right.$ & ysis of Variance: $p$ & $0.01)$ & & & \\
\hline
\end{tabular}

Table 5: Result of multiple-linear regression in TW distance

\begin{tabular}{|c|c|c|c|c|c|c|}
\hline & \multirow{2}{*}{ Item } & \multirow{2}{*}{$\begin{array}{l}\text { Partial regression } \\
\text { coefficient }\end{array}$} & \multirow{2}{*}{$\begin{array}{l}\text { Standardized partial } \\
\text { regression coefficient }\end{array}$} & \multirow{2}{*}{$p$ value } & \multicolumn{2}{|c|}{$95 \%$ Confidence interval } \\
\hline & & & & & Lower bound & Upper Bound \\
\hline \multirow{3}{*}{1} & Constant & 24.547 & & $p=0.000$ & 23.276 & 25.818 \\
\hline & TD distance & 0.130 & 0.180 & $p<0.001$ & 0.088 & 0.172 \\
\hline & $\left(\mathrm{R}^{2}=0.033, \mathrm{~A}\right.$ & sis of Variance: $p$ & $0.01)$ & & & \\
\hline
\end{tabular}

was created, the TD distance was chosen as the explanatory variable.

\section{Discussion}

When opening the door, this study was conducted to find out what is affected by the standing position. We hypothesized that the standing position when opening the door is affected by the surrounding circumstances such as the type of the door and the wall presence next to the door. We also predicted that it may be affected by physical factors such as a height.

From the results of this study, it is clear that the TD distance is adjusted according to the type of door. The door opening movement was performed from the position closer to the door in the sliding door condition than in the opening door condition.

It was also revealed that the TW distance was influenced not only by the presence of the wall beside the door but also by the type of the door. It was revealed that the door opening movement is performed in the standing position where the person stands away from the wall when the wall is present besides the door. And it was revealed that the physical features do not affect the TW distance. From the result of multiple regression analysis, when the standing position is away from the door, door opening movement is performed in the standing position away from the wall.

In this research, we defined the moment of holding the door handle as the door opening movement. However, the door opening movement is one frame in a series of movements of opening and passing through the door. Therefore, the door opening movement is considered to be influenced by the strategy of the next movement. A push open door was used under the hinged door condition in this study. It is considered that the door was opened with the human body accelerating forward and then passing through the door. In other words, the center of gravity moves forward during all of the door opening movement which is included after passing through the door. On the other hand, even if the human body accelerates forward until approaching the door, they have to stop to open the door sideways under the sliding door condition. The difference of the direction to open the door between the hinged door and the sliding door makes a difference of the standing position.

The wall presence affects the door opening movement, and it is considered that there was a strategy to open the door unconsciously away from the wall, being influenced by the wall presence. Under the sliding door condition, the number of the combination between right hand holding the door handle and left foot forward without wall presence was greater than the number of that with wall presence. In this condition, it would 
be considered that the center of gravity moved left to right. The difference of the standing position was little between these conditions, but it was suggested that the strategy to adapt the next movement was chosen.

The standing position we investigated in this study is considered to be a place where the force to open the door is applied to the floor. Therefore, the data obtained from this research can be applied to the installation position of a non-slip mat etc. in the situation where the automatic door cannot be installed. In addition, we think that there is a possibility that it can be applied to future architectural plans for the installation of doors in the future. However, in order to apply it to architectural planning, it is necessary to consider the standing position in the door opening movement of not only young healthy people but also elderly people. In addition, it is considered that clarifying the difference between healthy young people and elderly people can be applied to guidance on door opening movement and prevention for falling in activities in daily life for elderly people and people with disabilities. Furthermore, there would be a possibility that this data can contribute to fall prevention of elderly people and people with disabilities.

There were some limitations in this study. We defined that TW distance and TD distance were the distance between the former foot and the wall/door. Since the difference between the distance to the wall is different between the case where the former foot is the right foot and the case where the former foot is the left foot, further consideration is necessary on this point. Because there were a few left-handed subjects in the previous survey, this study targeted right-handed people. In the results of this research, the hand holding the handle was mainly used as the dominant hand, but it is necessary to further examine whether the right hand was used as the motion strategy or the right hand was used as the dominant hand. In addition, we used an open door and a sliding door that open from left to right in this research. It is necessary to analyse the relationship between the hand holding the handle and the former foot with other types of the doors, such as pull open hinged door, right to left open door and so on, in further studies.

\section{Conclusion}

We analysed the door opening movement of 93 young healthy volunteers and examined the distance between the great toe of the former foot and the door/wall (TD/TW distance) and the relationship between the surrounding environment and the physical features under the conditions of sliding doors and hinged door.

There were significant differences between TD and TW distance depending on door type, and wall presence showed significant difference in TW. TW distance and the height were significant predictors of TD distance. TD distance was a significant predictor of TW distance.

The results showed that the surrounding environment and the physical features affected the door opening movement. Data showed TD distance under the sliding door condition was significantly less than that under a hinged door condition, and this suggested that people might need to get closer to the door when opening the sliding door.

\section{References}

Japanese Ministry of the Environment. Hot spring usage situation. https://www.env.go.jp/nature/onsen/pdf/2-4_p_1.pdf.

Kurioka, H. and Funakawa, Y. (2004). Study on the moving distance when opening and closing the door, $A M J, 67-69$. (in Japanese)

Yamada, K. and Miyazaki, H. (2008) A study on town planning from the viewpoint of regional charcteristics in hot spring area: Trend of regional characteristics from point of geographical condition and process of formation. Journal of Architecture and Planning, Vol. 73, No. 626, 819-826. (in Japanese)

Okuma, K., Tsushima, E., Hasegawa, I., and Tsushima, H. (2001) Do age, gender, height and weight affect the result of Functional Reach Test? Annual Report of the Tohoku Section of Japanese Physical Therapy Association, Vol. 13, 1418. (in Japanese)

(Received May 14, 2019; accepted May 31, 2019) 